\title{
The Epiphany of Post-COVID: A Watershed for Pediatric Dentistry
}

\author{
Aishwaraya Gupta ${ }^{1}$, Syed G Shah ${ }^{2}$, Bhavna Kaul ${ }^{3}$, Nanika Mahajan ${ }^{4}$, Rakesh K Gupta ${ }^{5}$
}

\begin{abstract}
Coronavirus disease-2019 (COVID-19) has spread as a pandemic throughout the world, posing a serious public health threat. Dentists appear to have a higher risk of COVID-19 exposure amongst Healthcare workers. This danger is even more magnified in pediatric dentistry since infected children typically have an asymptomatic, mild, or severe clinical viral infection and so can considerably contribute to community-based COVID-19 transmission. These are unprecedented times that require unprecedented efforts with dynamic protocols to be adopted. It is very challenging, but also an amazing opportunity to UNLEARN, RELEARN, AND REORGANIZE our approach of dental practice, so as to adapt and come at par to the emerging "NEW NORMAL". Coronavirus-"THE ENFORCER" has enforced upon us, screening of patients for COVID-19, utilization of stringent disinfection \& sterilization protocols, be attired with personal protection such as PPE, N95 Mask, face shield, etc., to minimize chances of contraction or cross-contamination with social distancing and appropriate hygiene practices as a new norm. It has further enlightened the concept of "Non-aerosol" or "Minimally invasive techniques" that will possibly guide our therapeutic choices not only in the immediate future but also in the long term. The purpose of this review is to offer a compendious abridgment of literature available on COVID-19 to give an insight on recommendations that have been published about pediatric dental practice during COVID-19 and lays a concrete emphasis on the paradigm shift in the actual in-practice treatment options, to adapt to the changing circumstances during the pandemic and the times to follow, opening scenarios of prevention and cure that are more sustainable, safe, and efficient.
\end{abstract}

Keywords: Aerosol-free dentistry, Atraumatic restorative technique, Coronavirus, COVID-19, Hall technique, Infection control, Minimal invasive dentistry, Pediatric dentistry, Silver diamine fluoride.

International Journal of Clinical Pediatric Dentistry (2021): 10.5005/jp-journals-10005-2080

\section{INTRODUCTION}

Coronavirus disease-2019 (COVID-19) upsurge has unleashed an unexpected global health crisis that has created a profound impact on our daily lives. Besides the loss of life, this burgeoning Coronavirus pandemic has wreaked havoc on health services, education, economy and social life making the whole world go to a standstill.

As rightly said by Jimmy Dean,

"I can't change the direction of the wind, but I can adjust my sails to always reach my destination."

So, it is the time to REFLECT, REVIVE, RELEARN, AND RESTART.

Post-COVID-19, a dentist's life will never be the same. The time, however, is ripe for change. We need to bring about the necessary changes in our approach to dental practice and maneuver new ways of safe treatment to get back on our feet, returning to the 'New normal'.

These may be tough calls, on the face of it; but it is only under extreme pressure, does a block of carbon becomes a diamond. Since every adversity harbors an opportunity, coronavirus, while being the reason behind almost all things dire in recent times, can also be the harbinger of a positive change in the field of dentistry, where the role of the dentist is not only limited to taking care of teeth.

Because the COVID-19 scenario is changing unpredictably on daily basis, Pediatric dentists must be aware and updated to help patients, be vigilant in order to reduce risk, and limit viral transmission and modify their way of dental practice post-COVID such that both dentists and patients have a greater level of safety. \begin{tabular}{l}
\hline${ }^{1-5}$ Department of Pedodontics and Preventive Dentistry, Indira Gandhi \\
Government Dental College and Hospital, Jammu Tawi, Jammu and \\
Kashmir, India \\
Corresponding Author: Nanika Mahajan, Department of Pedodontics \\
and Preventive Dentistry, Indira Gandhi Government Dental College \\
and Hospital, Jammu Tawi, Jammu and Kashmir, India, Phone: \\
+919419260565, e-mail: drnanikamahajan@gmail.com \\
How to cite this article: : Gupta A, Shah SG, Kaul B, et al. The Epiphany \\
of Post-COVID: A Watershed for Pediatric Dentistry. Int J Clin Pediatr \\
Dent 2021;14(6):802-811. \\
Source of support: : Nil \\
Conflict of interest: : None
\end{tabular}

Overview of (CO)rona (VI)rus (D)isease (COVID-19)

Coronaviruses (CoV) are members of the coronaviridae family of viruses. COVID-19 disease is caused by a new beta coronavirus, SARSCoV-2, belonging to the subgenus sarbecovirus, Orthocoronavirinae subfamily, seventh member of the coronavirus family that infects humans, with most probable origin being the Chinese horseshoe bat. ${ }^{1}$ It is a spherical or elliptical shaped positive-stranded RNA virus having a diameter of $60-140 \mathrm{~nm}$. It consists of an envelope containing spike glycoproteins which gives it a characteristic crownlike appearance (coronam Latin term for crown). ${ }^{2,3}$

\section{EPIDEMIOLOGY}

Several coronaviruses have been linked to respiratory illnesses, such as SARS in 2002, H1N1 influenza in 2009, and MERS

(OTheAuthor(s). 2021 Open Access This article is distributed under the terms of the Creative Commons Attribution 4.0 International License (https://creativecommons. org/licenses/by-nc/4.0/), which permits unrestricted use, distribution, and non-commercial reproduction in any medium, provided you give appropriate credit to the original author(s) and the source, provide a link to the Creative Commons license, and indicate if changes were made. The Creative Commons Public Domain Dedication waiver (http://creativecommons.org/publicdomain/zero/1.0/) applies to the data made available in this article, unless otherwise stated. 
in 2012. A novel kind of coronavirus that causes pneumonia was first detected in December 2019 in Wuhan, Hubei Province, China. ${ }^{4}$ Initially, it was called as 2019 novel coronavirus (2019-nCoV). It was called novel coronavirus because it was a new strain of virus being discovered. This virus was referred to as SARS-CoV $-2^{5}$ by the International Committee on Taxonomy of Viruses and the disease was officially named COVID-19 by the World Health Organization (WHO). ${ }^{6}$ It was given the scientific name SARS-CoV-2 because the genome sequence of this virus features a close resemblance with SARS-CoV.

On January 31, 2020, ${ }^{7}$ the WHO declared the COVID-19 outbreak a Public Health Emergency of International Concern, and on 11th March 2020, it declared it a Pandemic. ${ }^{8}$ Recently, multiple variants of this virus have also been documented globally. The first variant was detected in UK in September 2020 and has spread across numerous countries. Other variants of this virus have also been detected in South Africa and Nigeria. ${ }^{9}$

\section{TRANSMISSION}

The mode of transmission of the disease is via airborne droplets generated from sneezing, coughing, and talking by an infected person; as a result of indirect contact with infected surfaces transmitting virus into the body through mucosal surfaces like the eyes, nose, and mouth or by direct contact with blood.

The average incubation period for human to human transmission is about 5 days, with an estimated range from 2 to
14 days. The virus is highly infectious, with basic reproduction number of 2-2.5, that is, each infected individual infects 2.5 healthy people on average in a susceptible population ${ }^{10}$ (Fig. 1).

\section{SYMPTOMS}

COVID-19 infection symptoms vary from being asymptomatic to severe pneumonia, acute respiratory distress syndrome, septic shock, and multi-organ failure, which results in death. ${ }^{4}$

Fever, chills, dry cough, sore throat, shortness of breath, and fatigue are common symptoms. Other symptoms include dysgeusia, hyposmia, nasal discharge or congestion, body aches, vomiting, diarrhea, hemoptysis, confusion, etc., COVID tongue or coated tongue has been also identified as a new symptom.

The majority of persons experience relatively mild symptoms. These individuals can serve as "carriers" as well as a reservoir for infection re-emergence. ${ }^{11}$

There is a high predilection of SARS-CoV-2 to bind to angiotensin-converting enzyme 2 (ACE-2) receptors in humans, present in various tissues and organs, mainly the lungs (Fig. 2). SARS-CoV-2 has been detected in the patients' blood and three main excretions (sputum, urine, and feces).

Moreover, men above the age of 56 , as well as people with comorbidities (hypertension, cardiovascular diseases, diabetes mellitus, immunosuppression, etc.), are more susceptible

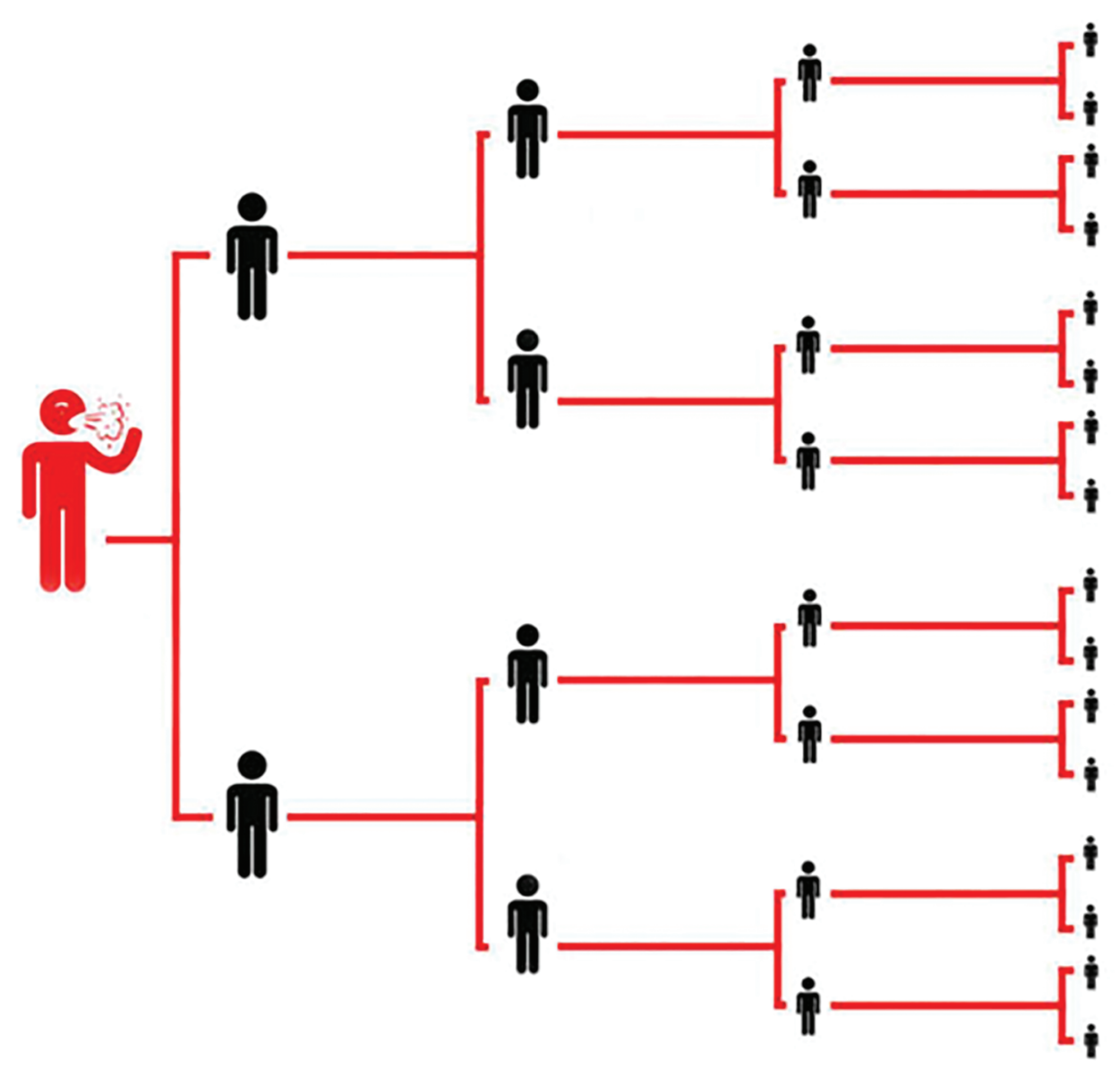

Fig. 1: Spread of COVID-19 


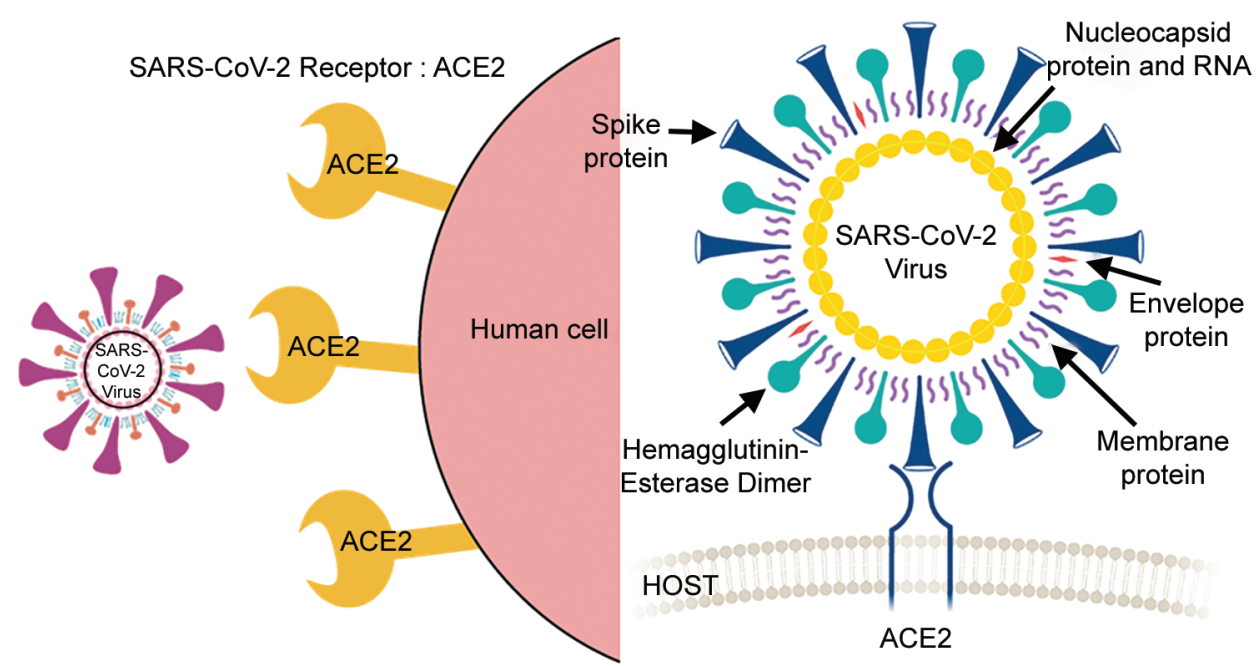

Fig. 2: SARS-CoV-2 predilection to bind to human angiotensin-converting enzyme 2 (ACE-2) receptors

to the disease and are reported to be high-risk groups for COVID-19 infection. ${ }^{4}$

There is a higher risk of contracting COVID-19 disease amongst Healthcare professionals who have daily physical contact with patients. Dental Healthcare workers are classified in a very high exposure risk category by the Occupational Safety and Health Administration (OSHA). ${ }^{12}$

\section{Diagnosis}

The diagnosis is made using a combination of the following factors:

- Epidemiologic evidence [travel history or interaction with a laboratory-confirmed case].

- Clinical manifestations.

- CT imaging.

- Laboratory investigations

- Virus detection

- RT-PCR

- Rapid antigen test

- Antibody detection. ${ }^{13}$

\section{Coronavirus Disease in Children}

Most children are asymptomatic or have minor, nonspecific symptoms. As a result, they may have a grave impact on community-based spread of COVID-19. Pediatric dentistry is more vulnerable to this risk and each individual should be considered as a potential carrier of COVID-19. Children's mortality seems to be uncommon, however, children having comorbidities are still a cause of concern.

It is still unclear why COVID-19 instances in children are less severe than those in adults. The possible reason could be that children's innate immune responses are more robust and active, they have fewer underlying diseases and have less exposure to air pollution and tobacco smoke as adults, thus having a stronger respiratory system. ${ }^{14}$ Moreover, SARS-CoV-2 has a lower capacity to elicit an immediate inflammatory response, ${ }^{15}$ structural and functional immaturity of ACE2 receptors in children offers low affinity to the pathogen ${ }^{10}$ resulting in improved outcome in children.

\section{Saliva - Reservoir for Coronavirus}

SARS-CoV-2 binds to human ACE-2 receptors, which are abundant in salivary glands; this might explain the occurrence of SARS-CoV-2 in secretory saliva.

\section{COVID-19 virus can occur in saliva by three distinct routes:}

- Virus may enter the oral cavity with liquid droplets that are regularly exchanged between them from the upper and lower respiratory tracts.

- COVID-19, which is found in the blood, can enter the mouth through the crevicular fluid.

- By infection of the major and minor salivary glands, via the salivary ducts with subsequent particle release into the saliva. ${ }^{16}$

\section{Why is Dentistry in Jeopardy?}

Dentistry, particularly, is at a greater risk of transmitting COVID-19 mainly because (Fig. 3);

- The work area is close to the patient's oropharyngeal region, which is thought to be the most prevalent habitat of virus liable for transmission.

- SARS-CoV-2 existence in secretory saliva. ${ }^{16}$

- Aerosols generated by mixing of water mist produced by air-driven high-speed hand piece, air polisher, etc., with patient's saliva and blood may remain suspended for long time in the air. ${ }^{17}$

- Dental procedures are time-consuming, resulting in the prolonged presence of aerosols across a vast area of the operatory, posing disease risk.

- By coming in contact with saliva, blood, or other patient related materials directly.

- Traditional protective measures are not fully safe.

- Contaminated dental equipment serve as a secondary source of infection.

- Both dental professionals and patients are at risk of becoming infected and spreading the virus through direct contact.

\section{General Recommendations for Dental Practice}

Prevention is Better than Cure!

It should be borne in mind that the virus and its math is novel and unpredictable. In this situation, the "Need of the hour is 


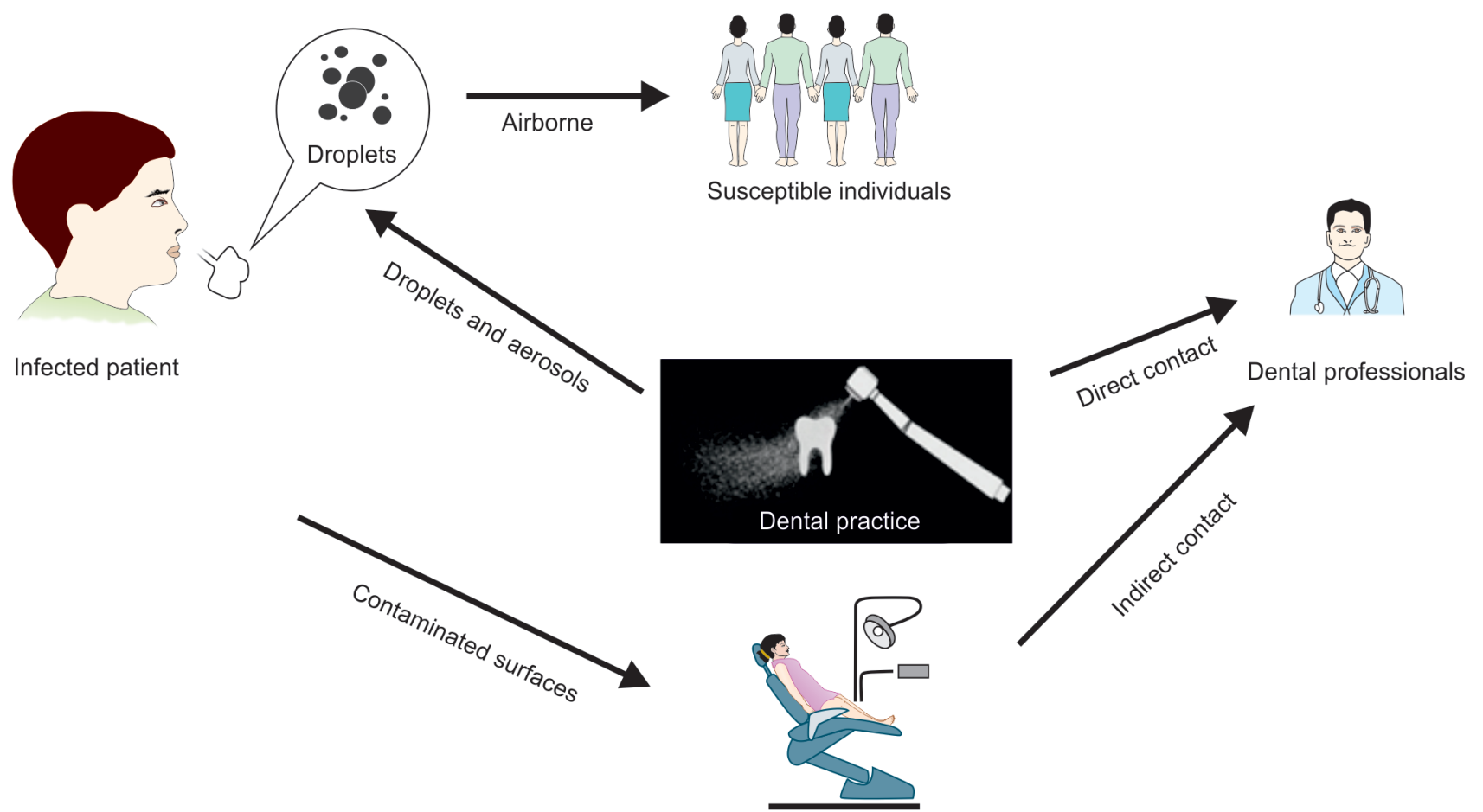

Fig. 3: COVID-19 transmission dynamics in dental care setting

Prevention". It is high time to take necessary precautions for our survival. Precautions for containment of virus in as well as through dental clinic include:

- Stage I-Preparative Stage

- Stage II- Execution Stage

- Stage III- Follow up and Review

\section{Stage I: Preparative Stage}

- Prophylaxis Against COVID-19 for Doctor and Healthcare Worker

- Before returning to work in the clinics, COVID-19 test must be done.

- Hydroxychloroquine Prophylaxis

For Healthcare professionals involved in the management of suspected or confirmed cases of COVID-19, the ICMR recommends using hydroxychloroquine for SARSCov-2 infection prevention except for patients with comorbidities. (Taking $400 \mathrm{mg}$ twice a day on the first day, then $400 \mathrm{mg}$ once a week for the next 7 weeks, with meals is recommended). ${ }^{18}$

- Criteria for Healthcare professionals with SARS-CoV-2 Infection to return to work-

- Fever relief without the use of medicines and

- Symptoms have improved (e.g., cough, breathlessness), and

- Negative results of SARS-CoV-2 RNA from at least two consecutive respiratory specimens taken 24 hours apart done via FDA-approved molecular viral assay. ${ }^{19}$

\section{- Dental Clinic ${ }^{20}$}

- Dental clinic ventilation and air quality monitoring-

- Let the natural air circulate throughout the clinic by keeping the windows open and using an exhaust blower.
- While performing procedures, avoid using a ceiling fan.

- Place the table fan behind the operator with airflow directed toward the patient. A strong exhaust fan positioned such that it generates a one-way flow of air away from the patient.

- While performing the procedure, the air conditioning system should preferably be switched off and the filters cleaned on a regular basis.

- An indoor portable air cleaning equipment with air filters and UV light can be utilized.

- The entrance, waiting area and reception area of the clinic-

- Post visual reminders regarding respiratory cleanliness, social distancing, cough etiquette, and waste disposal at the clinic's entrance.

- At the front desk, install a glass or plastic barrier.

- Ascertain that enough three-layer masks, sanitizers, and paper tissues are available at the registration desk.

- Waiting chairs should be at least 6 feet apart.

- Any places must be clear of all contaminates / fomites such as periodicals, toys, television remotes, etc.

- Limit the number of people sitting in the waiting area.

- Cashless/contactless/online payment should be adopted.

- Training and education of Healthcare Workers (HCW).

- Sterile Isolated or negative pressure room for carrying out treatment protocols.

- Exclusive place for donning and doffing of PPE.

- Separate designated place for sterilization.

- Sensor taps or elbow handle taps in restrooms. Paper towels should be used.

- Accessibility of PPE to HCW.

- Environmental Infection Control -

- After treating a suspected or confirmed COVID-19 patient, wait 15 minutes before disinfecting the dental operatory. 
- Disinfection of the Surfaces and the Environment;

- Floors: Cleaning in two steps (Detergent and 1 percent sodium hypochlorite, freshly made with a 10-minute contact period).Mop the floor from the far corner of the room to the door. Frequency: Every 2 hours or after each patient or large spill.

- The remaining surfaces: 1 percent sodium hypochlorite, freshly prepared to be used for a contact period of 10 minutes. Dust in straight, overlapping lines with damp dust. Frequency: Before and after work on daily basis.

- Delicate Electronic equipment: Before each patient interaction, an alcohol-based rub/spirit (60-90\% alcohol) swab should be used to clean the equipment.

- Fogging: This technique is utilized for "no-touch surface disinfection" and air disinfection after a broad area has been contaminated. Prepare a $20 \%$ working solution of 0.01 percent silver nitrate and 11 percent hydrogen peroxide $(\mathrm{w} / \mathrm{v})$ solution. The duration of fogging is generally 45 minutes, accompanied by an hour of contact time/dwell time. The room may then be opened, and fans can be turned on to provide aeration. Use a sterile or clean towel, dry/clean wet surfaces.

\section{- Hand Hygiene}

- Practice strict hand hygiene as per WHO guidelines. ${ }^{21}$

- At beginning and after all patient interaction, contact with potentially infected materials, and before and after putting on and taking off gloves or PPE.

- Wash hands with soap and water for at least 20 seconds or use an alcohol-based hand rub with 60-95 \% alcohol.

\section{Stage II- Execution Stage}

\section{- Telescreening/Teleconsultation}

Telephone triage and screening are recommended. Dentists should speak with parents over the phone to acquire a complete dental history to know whether there is an emergency or try to manage problems with the $3 \mathrm{~A}$ approach- Advice, Analgesics, and Antimicrobials where necessary. ${ }^{21}$ The adoption of alternate modalities for patient interactions, such as video-calling, should be fostered.

\section{- Patient Selection Criteria During the COVID-19 Outbreak}

It is mandatory to prioritize and categorize the oral conditions / ailments during the prevailing pandemic before considering for treatment (Fig. 4).

\section{- Patient Management Protocol in the Clinic Area ${ }^{21}$}

- Patient evaluation-

- The temperature of both the child and their caregiver is recorded using infra-red thermometers.

- Conduct an investigation of travel history to any area afflicted by the COVID-19 outbreak within 14 days preceding the dental appointment, any history of contact, and also screen for any symptoms of COVID-19. ${ }^{22}$

- The child should be accompanied by only one attendant.

- Masks and shoe covers should be provided to them. Young children under the age of two should not use facemasks.

- The kid and the caregiver should wash their hands with soap and water or use alcohol based hand rub.
- Practice nonaerosol generating procedures (AGPs). Use of hand instruments in conjunction with chemo mechanical caries removal should be emphasized. ${ }^{23}$

- Triple layer surgical mask, protective eyewear/face shield, and gloves should be used for appointments that do not result in aerosols and need examination.

- High-speed hand pieces, slow hand pieces, 3-in-1 syringe, Ultrasonic scalers, etc., generate aerosols. Aerosol-generating procedure should be arranged as the last appointment of the day. ${ }^{23}$

- Use N95 respirators and surgical masks to shield the wearer's face from airborne particles and liquid contaminants. $\mathrm{NIOSH}$-approved $\mathrm{N} 95$ respirators provide an extremely close face fit and effective filtration of at least $95 \%$ of airborne particles. The respirator's edges are meant to establish a seal around the nose and mouth. It is not recommended to use a respirator with a valve because it allows infectious droplets to escape. ${ }^{24}$

- PPE should be worn while dealing with high risk and very high-risk procedures.

- N95 masks can be covered with a surgical mask to extend their shelf life, and only the surgical mask should be discarded after usage.

- Ten or 11 o'clock position is recommended for working. Eight o'clock position should be avoided to prevent splatter. ${ }^{23}$

- About $0.5-1 \%$ hydrogen peroxide or $0.2 \%$ povidine-iodine should be used as pre procedural mouth wash provided the child can rinse and not swallow. Recent studies indicate that chlorhexidine is ineffective against the SARS-CoV-2 virus since it mainly acts against bacteria and not viruses. ${ }^{25}$

- The use of rubber dam is advised because they decrease airborne particles by $70 \%$ in an approximate three-foot diameter working field. ${ }^{23}$

- High volume suction and four-handed dentistry is recommended. ${ }^{23}$

- To avoid cross infection, use antiretraction hand pieces, antiretraction valves and retrograde aspiration. ${ }^{26}$

- The use of an Intraoral X-ray is not suggested since it might cause salivation and coughing. Extra oral dental radiographs, such as panoramic radiography and cone-beam computed tomography (CBCT), should be used instead. ${ }^{1}$

- During the procedure, only those people who are necessary for patient treatment should be present.

- One percent sodium hypochlorite is used for disinfection of Dental Chair and other auxiliary parts within a 3-foot radius with a clean, sterilized cotton/gauge piece, utilizing an innerto-outer surface technique, leaving it to dry. For each surface, a new piece of cotton or gauge should be used.

- Clean the hand piece with a hand piece cleaning solution before autoclaving.

- Before pouring impressions, disinfect them using a suitable disinfectant.

- Sodium hypochlorite disinfectant solution should be flushed for 30-40 seconds through all water pipelines, three-in-one syringes, water outlets, etc.

- Medical waste generated from treating confirmed or suspected COVID cases must be placed in "gooseneck" ligated, yellow colored, double-layered medical waste package bags and should be disposed according to the medical waste disposal regulations. ${ }^{23}$ 


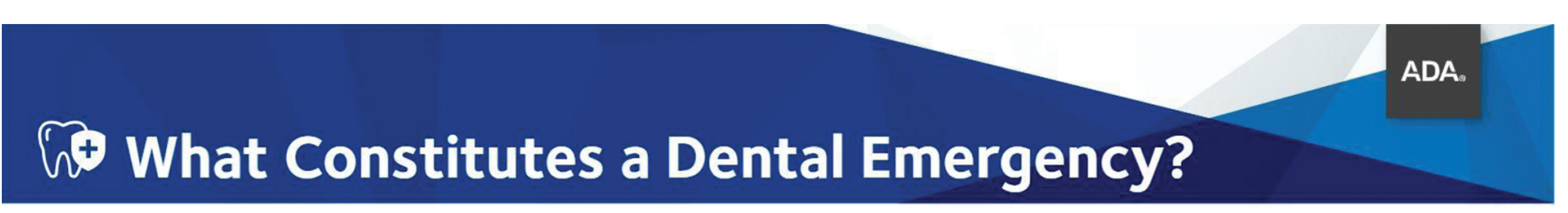

The ADA recognizes that state governments and state dental associations may be best positioned to recommend to the dentists in their regions the amount of time to keep their offices closed to all but emergency care. This is fluid situation and those closest to the issue may best understand the local challenges being faced.
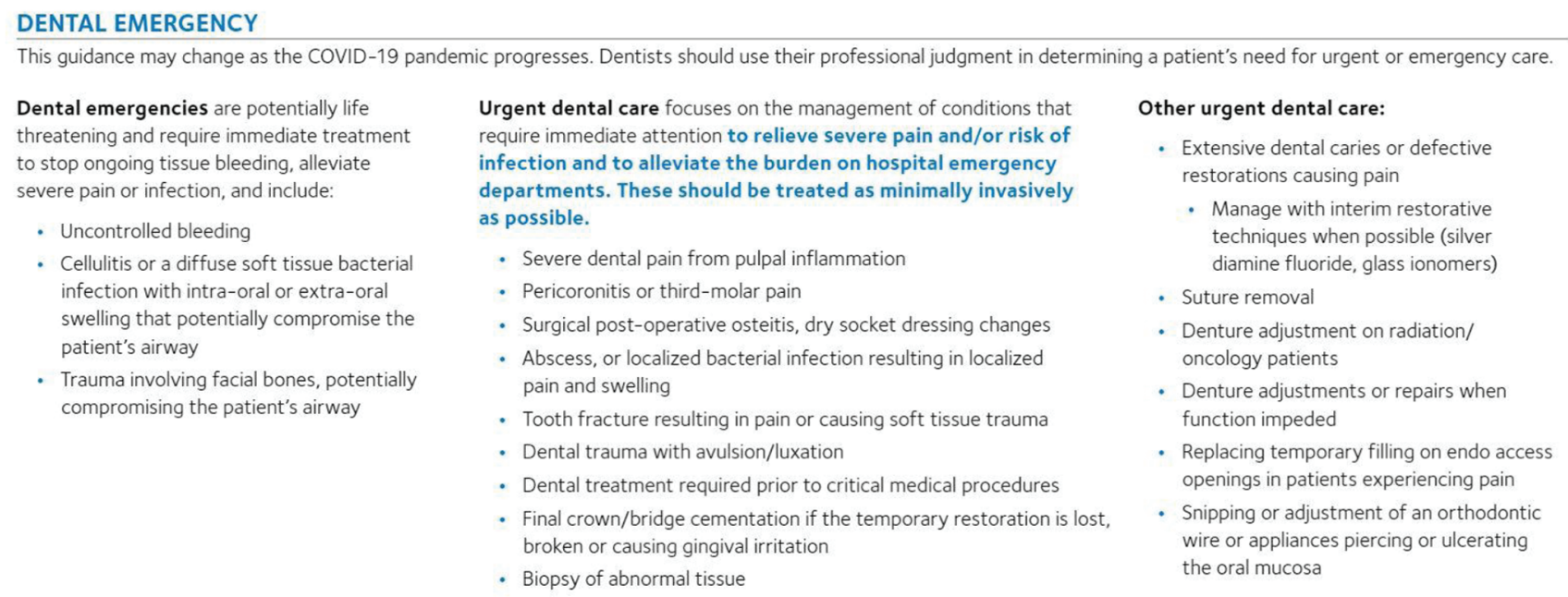

\section{DENTAL NON EMERGENCY PROCEDURES}

Routine or non-urgent dental procedures includes but are not limited to:

- Initial or periodic oral examinations and recall visits, including routine radiographs

- Routine dental cleaning and preventive therapies

- Orthodontic procedures other than those to address acute issues (e.g. pain, infection, trauma) or other issues critically necessary to prevent harm to the patient

Updated $3 / 31 / 20$

Fig. 4: Categorization of dental treatment needs by ADA

\section{Stage III - Follow-up and Review}

After 24 hours and after one week, the patient should be contacted by phone to check whether he has acquired any symptoms.

\section{Oral Health Prevention during COVID-19 Pandemic}

- It is difficult to maintain regular dental check-ups during COVID-19, necessitating an emphasis on preventive measures and maintenance of good oral health through oral health education interventions, dental flossing, at-home oral hygiene methods, and low cariogenic diet, interruption of incorrect alimentary habits and physical activity of the child must be closely monitored at home to avoid dental trauma.

- Good dental health must be paired with a healthy lifestyle in order to contribute to good overall health.

- At-home Care for the Children During Pandemic

- For nonpainful earlier carious lesions on deciduous or permanent teeth;

- Cavity should be kept free of any food particles.

- Avoid too hot/ cold foods.

- Over retained deciduous teeth can be managed by advising hard consistency food which may facilitate the mechanical exfoliation of that tooth.

\footnotetext{
- Extraction of asymptomatic teeth

- Restorative dentistry including treatment of asymptomatic carious lesions

- Aesthetic dental procedures
}

FOR THE LATEST UPDATES, VISIT ADA.ORG/VIRUS
- For removable orthodontic appliance, parents must make sure that the child takes adequate hand hygiene precautions before placing the appliance into the mouth, as well as properly cleans the appliance.

- When using fixed orthodontic devices, parents should ensure that their child does not consume any hard or viscous food.

\section{Paradigm Shift in Clinical Recommendations in Post- COVID-19 Era}

In children, the capriciousness of COVID-19 infection, their carrier status and difficulty in diagnosing symptoms, presents a challenge for all pediatric dentists worldwide. AGPs should be minimized feasibly, the use of Minimal Invasive Techniques as alternatives for caries management should be done.

\section{- Behavior Management}

- Children might be frightened by dentists dressed in full PPE. Euphemism of PPE as a space suit of an astronaut can be done (Fig. 5A).

- Whenever possible, put on the protective equipment in front of the child making him explain the use of this equipment.

- Also, over the PPE, a sticker of health worker's photograph (that can be disinfected easily) can be displayed so that child can identify the dentist inside the PPE (Fig. 5B). 

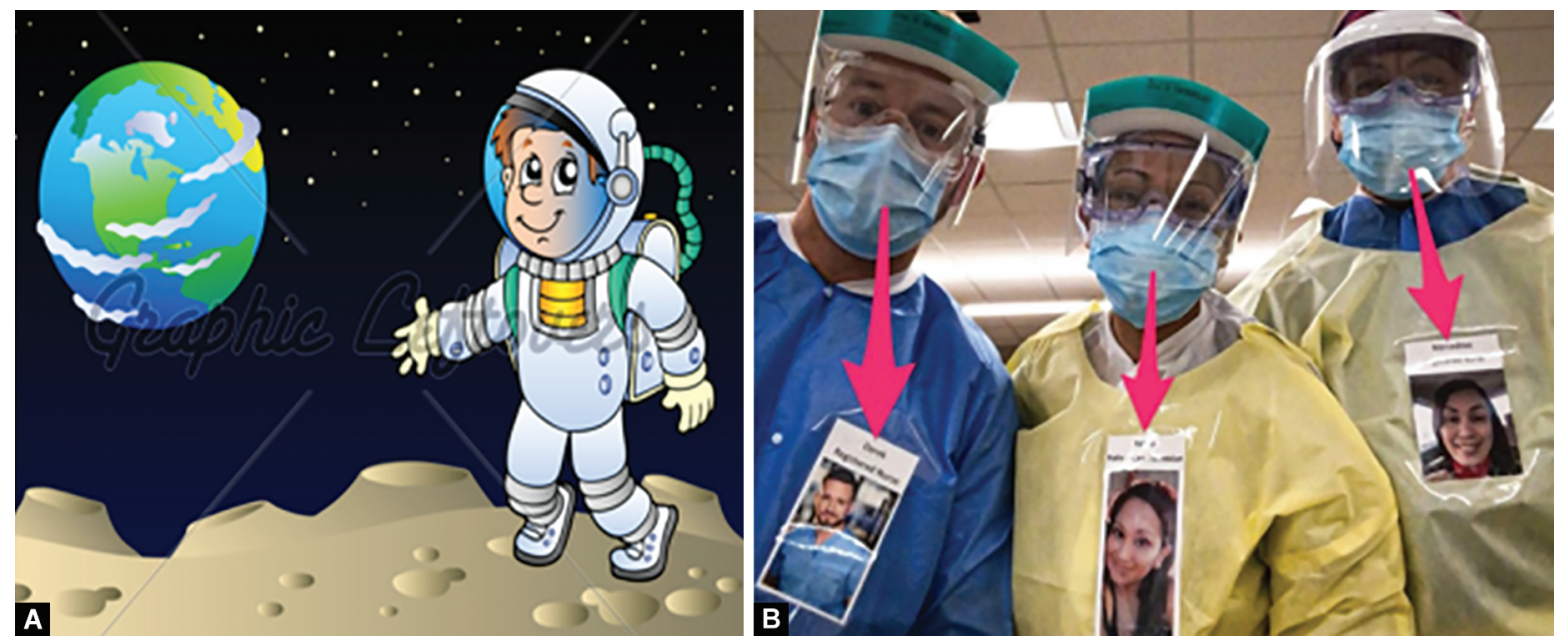

Figs 5A and B: (A) Euphimism of dentist in PPE as an astrounaut; (B) Sticker with dentist's photograph displayed over the PPE

- The face masks can have the designs of a cartoon mouth; the face shields can also incorporate interesting designs to make them less fearful for the patient.

- Proper communication between the pediatric dentists, parents and children, as well as appropriate coping strategies are essential. Also, videos and examples of what to expect; $P P E$, etc., to make them familiar with the new protocols.

- The AAPD suggests allocating specific hours in the day for patients who require physical behavior management, treating children while sitting on their parent's lap, provided that parent is wearing a mask and has passed negative screening requirements. ${ }^{27}$

\section{- Biological Caries Management Techniques}

Dental caries is largely preventable. Therefore, it is only logical to emphasize on preventive and therapeutic strategies for oral health and also prioritize the concept of Safer Aerosol-Free Emergent Dentistry (SAFE Dentistry) during this time.

- Ozone therapy can be used as a treatment option for caries management

- Alternatives to biological therapy for teeth that are asymptomatic or show symptoms of reversible pulpitis include:

- Atraumatic Restorative Technique (ART)

- The World Health Organization (WHO) has recommended ART as a method of treating and preventing caries in people who do not have access to traditional dental treatment. ${ }^{28}$

- The ART method entails removing infected dentine with hand instruments, then placing a restorative material and sealing adjacent pits and fissures simultaneously with high viscosity glass-ionomer placed under finger pressure.

- Both primary and permanent teeth can be treated with ART to manage caries successfully. There is a high-cariespreventive effect of ART sealents. ${ }^{29}$

- As a result, in the post-COVID-19 age, ART appears as a useful, evidence-based alternative to traditional techniques for treating and preventing carious lesions.

- Interim Therapeutic Restorations (ITR)

- As defined by AAPD, Interim therapeutic restoration uses procedures similar to ART but has distinct therapeutic objectives. In young, noncooperative patients, or patients with special healthcare needs, ITR aids in the restoration of caries and prevention of further decalcification.
- When dental treatment needs to be postponed or when traditional cavity preparation and/or insertion of conventional dental restorations are not possible, this technique can be employed. ${ }^{30}$

- Step-by-step excavation of multiple open carious lesions before final restoration is a modified use of ITR. ${ }^{31}$

- The ITR technique comprises of removing caries by use of hand or slow speed rotary instruments avoiding pulp exposure. Restoration leakage can be minimized by removing as much caries as possible from the periphery of the lesion and restoring the tooth with an adhesive restorative material such as self-setting or RM-GIC. ${ }^{32}$

\section{- Chemo Mechanical Caries Removal}

- It is a more recent noninvasive method that uses a chemical agent for removing carious infected dentin.

- This method relies on dissolution to eliminate caries. Rather than drilling, this technique removes soft carious structure with a chemical agent and an atraumatic mechanical force.

- In this, a solution/gel is applied to the carious dentin, enabling it to soften the tissue before scraping it away with specialized hand instruments. Carisolv and Papacarie are the most in-demand agents right now. ${ }^{33}$

- Therefore, the use of chemomechanical method for caries removal is a potentially useful dental tool in the present COVID-19 epidemic and beyond.

\section{- The Hall Technique}

- The Hall technique (HT) is a child-friendly non-surgical/noninvasive technique for restoring a carious but asymptomatic and non-septic primary molar.

- HT is based on the use of a prefabricated metal crown (PMC) and GIC to seal non-pulpally involved carious lesions on primary molars that do not show signs of irreversible pulpitis. ${ }^{34}$

- This technique involves placement of prefabricated metal crown on the affected molar after creating space via orthodontic spacers/separators, which are applied for 3-5 days. There is no use of local anesthesia, and the carious tissue is not removed surgically. ${ }^{35}$

- HT is currently regarded as "the golden standard" for treating non pulpally involved multi surface carious primary molars. ${ }^{36}$

- HT is highly cost-effective, simple and quick technique to be used in children since it avoids injections and drilling and can also be used for anxious children. ${ }^{34}$ 
- The HT also offers a therapeutic option to maintain poorly broken down first permanent molars (FPMs) symptom free until the optimal age for extractions. ${ }^{37}$

- Hypo mineralized or hypo plastic FPMs with caries or sensitivity can be managed for short-term using $\mathrm{HT}^{38}$

- HT provides an alternative to drilling, minimizing aerosol production in the post-COVID-19 era.

\section{- Silver Diamine Fluoride (SDF)}

- Silver Diamine Fluoride is a colorless, odorless liquid containing silver, fluoride, and ammonia. These silver ions in SDF kill the bacteria causing the cavity.

- Indications include arresting caries in high caries-risk children, molar incisor hypo mineralization of first permanent molar, desensitization of non-carious tooth lesions, and for, uncooperative, medically compromised children or children with special Healthcare needs ${ }^{35}$ (Fig. 6).

- $38 \%$ SDF solution (applied biannually) is preferred over $5 \%$ $\mathrm{NaF}$ varnish by the clinicians. ${ }^{39}$

- The limitation is that it causes blackish discoloration of carious tooth structure.

- It is contraindicated in patients with silver allergy or when caries has approached the pulp.

- No serious adverse effects have been reported. ${ }^{40}$

- It is cost-effective as one drop can be used for multiple teeth.
- Thus, SDF, the silver bullet of dentistry is a safe, non-invasive, painless alternative to traditional cavity drilling procedures.

\section{Conclusion}

"A shift in our perception and interpretation enables us to break old habits and awaken new possibilities and opportunities for balance, healing, and transformation."

In these unforeseen times, events are unfolding rapidly. Newer insights are emerging daily and the biggest question that lies ahead is "What next?." Hence, all dentists must be cognizant of recent developments and recommendations by state, federal, national, and international bodies so that necessary measures can be taken to mitigate contagion. As the world is gripped in fear amidst COVID-19 pandemic, dentists should also take care of their mental health and should stay proactive, calm, and free of stress and prepared to be in a better capacity to fulfill their roles and provide patients with the best dental care.

COVID-19 has had a significant influence on pediatric dental practice and will continue to do so. It paved a way for the advent of new approaches and management strategies in pediatric dentistry. It has also brought to light the Dentist's new role in primary Healthcare by including dentists to assist with COVID vaccination administration forthcoming. It is an amazing opportunity to bring out reform in dental practice.
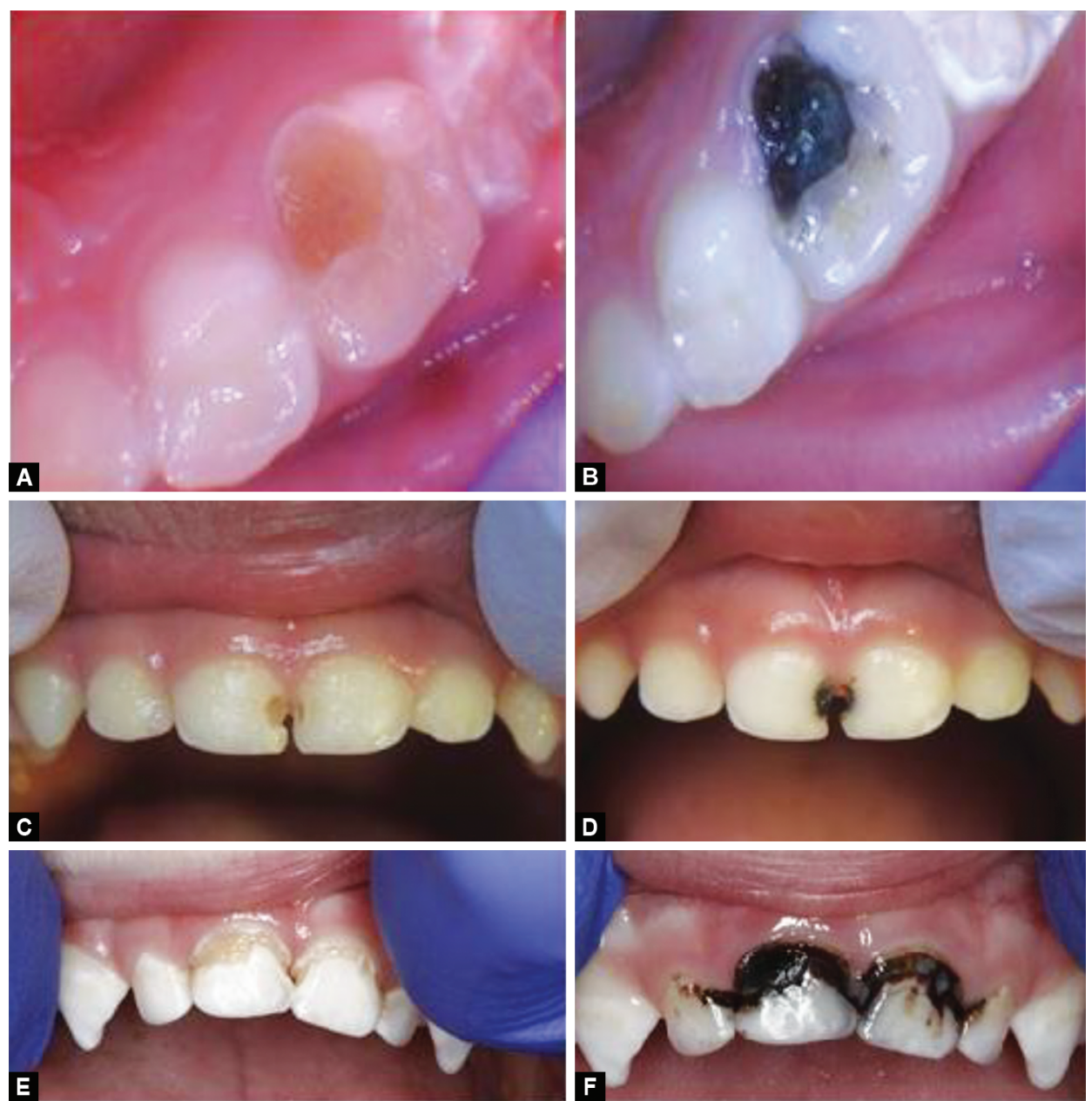

Figs 6 A to F: Treatment with SDF 
During the pandemic, smart technology systems bloomed into the most powerful remote communication tool for providing oral health education and motivation to children and parents about preventative measures and home care management.

Pediatric dentists, on the other hand, have strengthened their commitment to practice by strictly adopting infection control protocols, improving and modernizing treatment approaches, implementing more prevention-focused practices and non-aerosol, atraumatic, minimally invasive techniques while managing pediatric patients.

TO CONCLUDE;

"UNLEARN, RELEARN, REORGANIZE, AND RESTART."

\section{References}

1. Meng L, Hua F, Bian Z. Coronavirus disease 2019 (COVID-19): emerging and future challenges for dental and oral medicine. J Dent Res 2020;99(5):481-487. DOI:10.1177/0022034520914246

2. Carlotti APCP, Carvalho WB, Johnston C, et al. COVID-19 diagnostic and management protocol for pediatric patients. Clinics (Sao Paulo) 2020;75:e1894. DOI: 10.6061/clinics/2020/e1894

3. Zhu N, Zhang D, Wang W, et al. China novel coronavirus investigating and research team. A novel coronavirus from patients withpneumonia in china, 2019. N Engl J Med 2020;382(8):727-733. DOI: 10.1056/NEJMoa2001017

4. Cascella M, Rajnik M, Cuomo A, et al. Features, evaluation, and treatment of coronavirus. 2020. In: StatPearls [Internet]. Treasure Island (FL): StatPearls Publishing; 2020.

5. International Committee on Taxonomy Viruses. Naming the 2019 Coronavirus. Available online: https://talk.ictvonline.org

6. World Health Organization. Novel Coronavirus (2019-nCoV) Situation Report-22. Available online: https://www.who.int/docs/defaultsource/coronaviruse/situation-reports/20200211-sitrep-22-ncov.pdf

7. World Health Organization. Statement on the second meeting of the International Health Regulations (2005) Emergency Committee regarding the outbreak of novel coronavirus (2019-nCoV). Available online: https://www.who.int/news-room/ detail/30-01-2020-statement-on-the-second-meeting-of-theinternational-health-regulations-(2005)-emergency-committeeregarding-the-outbreak-of-novel-coronavirus-(2019-ncov)

8. World Health Organization. WHO Director-General's opening remarks at the media briefing on COVID-19-11 March 2020. Available online: https://www.who.int/director-general/speeches/who-directorgeneral-s-opening-remarks-at-the-media-briefing-on-covid-19--11-march-2020

9. Centers for Disease Control and Prevention, New COVID-19 variants Updated Jan. 9, 2021. Available online: https://www.cdc.gov/ coronavirus/2019-ncov/transmission/variant.html

10. Paglia L. COVID-19 and paediatric dentistry after the lockdown. Eur J Paediatr Dent. 2020;21(2):89. DOI: 10.23804/ejpd.2020.21.02.01

11. Parihar A, Saxena A, Rawat A, et al. COVID - 19: influenced alteration in dental OPD management. Int J Appl Dent Sci 2020;6(2):349-355.Available online at https://www.oraljournal. com/pdf/2020/vol6issue2/PartF/6-2-43-203.pdf

12. Centers for Disease Control and Prevention, Interim Infection Prevention and Control Guidance for Dental Settings During the Coronavirus Disease 2019 (COVID-19) Pandemic. Updated Dec. 4, 2020. https://www.cdc.gov/coronavirus/2019-ncov/hcp/dentalsettings.html

13. World Health Organization (WHO). Diagnostic testing for SARS-COV-2. Interim guidance. 11 September 2020. Available from: https://www. who.int/publications/i/item/diagnostic-testing-for-sars-cov-2

14. Lee PI, Hu YL, Chen PY, et al. Are children less susceptible to COVID-19? J Microbiol Immunol Infect 2020;53(3):371-372. DOI: 10.1016/j. jmii.2020.02.011
15. Park JY, Han MS, Park KU, et al. First pediatric case of coronavirus disease 2019 in Korea. J Korean Med Sci 2020;35(11):e124. DOI: 10.3346/jkms.2020.35.e124

16. Sabino-Silva R, Jardim ACG, Siqueira WL. Coronavirus COVID-19 impacts to dentistry and potential salivary diagnosis. Clin Oral Investig 2020;24(4):1619-1621. DOI: 10.13039/501100000024

17. Wei J, Li Y. Airborne spread of infectious agents in the indoor environment. Am J Infect Control 2016;44(9 Suppl):S102-S108. DOI: 10.1016/j.ajic.2016.06.003

18. Ministry of Health and Family Welfare, Government of India, Advisory on the Use of Hydroxy-Chloroquine as Prophylaxis for SARS-CoV-2 Infection, Ministry of Health and Family Welfare, Government of India, New Delhi, India, 2019. Available online: https://www.mohfw.gov.in/pdf AdvisoryontheuseofHydroxy chloroquinasprophylaxisforSARSCoV2infection.pdf

19. Centers for Disease Control and Prevention, Criteria for Return to Work for Healthcare Personnel with SARS-Cov-2 Infection (Interim Guidance). Updated Aug. 10, 2020. Available online: https://www. cdc.gov/coronavirus/2019-ncov/hcp/return-to-work.html

20. Guidelines for Dental Professionals in Covid-19 pandemic situation Ministry of Health and Family Welfare. Issued on 19/05/2020.

21. World Health Organization. Clean hands protect against infection. Available online: https://www.who.int/gpsc/clean_hands_ protection/en

22. Symptoms of coronavirus. National Center for Immunization and Respiratory Diseases (NCIRD), Division of Viral Diseases. Centers for Disease Control and Prevention. Updated May 8, 2020. https: //www.cdc.gov/coronavirus/2019-ncov/symptoms-testing/ symptoms.html

23. Peng $X, X u X, L i$, et al. Transmission routes of 2019-nCoV and controls in dental practice. Int J Oral Sci 2020;12(1):9. DOI: https:// doi.org/10.1038/s41368-020-0075-9

24. N95 Respirators, Surgical Masks, and Face Masks-FDA. Available online: https://www.fda.gov/medical-devices/personal-protectiveequipment-infection-control/n95-respirators-surgical-masks-andface-masks

25. Ather A, Patel B, Ruparel NB, et al. Coronavirus disease 19 (COVID-19): implications for clinical dental care. J Endod 2020;46(5):584-595. DOI: 10.1016/j.joen.2020.03.008

26. Swaminathan $Y$, Thomas J. "Aerosol" - a prospective contaminant of dental environment. IOSR J Dent Med Sci 2013;11(2):45-50. DOI: 10.9790/0853-1124550

27. American Academy of Pediatric Dentistry 2020. A guide for re-entry into practice for pediatric dentists during the COVID-19 pandemic. https://www.aapd.org/about/about-aapd/news-room/covid-19/

28. Tedesco TK, Calvo AF, Lenzi TL, et al. ART is an alternative for restoring occlusoproximal cavities in primary teeth - evidence from an updated systematic review and meta-analysis. Int J Paediatr Dent 2017;27(3):201-209. DOI: 10.1111/ipd.12252

29. De Amorim RG, Frencken JE, Raggio DP, et al. Survival percentages of atraumatic restorative treatment (ART) restorations and sealants in posterior teeth: an updated systematic review and metaanalysis. Clin Oral Investig 2018;22(8):2703-2725. DOI: 10.13039/ 501100003593

30. Dhar V, Marghalani AA, Crystal YO, et al. Use of vital pulp therapies in primary teeth with deep caries lesions. Pediatr Dent 2017;39(5):146159. Erratum in: Pediatr Dent 2020;42(1):12-15. PMID: 29070150.

31. Coll JA, Campbell A, Chalmers NI. Effects of glass ionomer temporary restorations on pulpal diagnosis and treatment outcomes in primary molars. Pediatr Dent 2013;35(5):416-421.PMID: 24290553

32. Berg JH. Glass ionomer cements. Pediatr Dent 2002;24(5):430438.PMID: 12412957

33. Ganesh M, Dhaval P. Chemomechanical caries removal (CMCR) agents: review and clinical application in primary teeth. J Dent Oral Hyg 2011;3(3):34-45. Available online at https://academicjournals. org/article/article1379416670_Ganesh\%20and\%20Parikh.pdf 
34. Welbury RR. the Hall Technique 10 years on: its effect and influence. Br Dent J 2017;222(6):421-422. DOI: 10.1038/sj.bdj.2017.262

35. Innes NPT, Chu CH, Fontana M, et al. A century of change towards prevention and minimal intervention in cariology. J Dent Res 2019;98(6):611-617. DOI: 10.1177/0022034519837252

36. Deery $C$. The hall technique: a paradigm shift in our care of children with caries. Dent Update 2015;42(10):903-904. DOI: 10.12968/ denu.2015.42.10.903

37. SDCEP 2018. Prevention and management of dental caries in children. https://www.sdcep.org.uk/wp-content/uploads/2018/05/ SDCEP-Prevention-and-Management-of-Dental-Caries-in-Children2nd-Edition.pdf
38. Millar LM, Cairns AM, Fowler L. Preformed metal crowns for the permanent dentition. Prim Dent J 2015;4(4):44-45. DOI: 10.1308/205016815816682281

39. Urquhart O, Tampi MP, Pilcher L, et al. Nonrestorative treatments for caries: systematic review and network meta-analysis. J Dent Res 2019;98(1):14-26. DOI: 10.1177/0022034518800014

40. Seifo N, Cassie H, Radford JR, et al. Silver diamine fluoride for managing carious lesions: an umbrella review. BMC Oral Health 2019;19(1):145. DOI: 10.1186/s12903-019-0830-5 